\title{
Electromagnetic production of hyperon resonances
}

\author{
K. Hicks, D. Keller and W. Tang \\ Dept. of Physics \& Astronomy, Ohio University, Athens $\mathrm{OH} 45701$
}

\begin{abstract}
The study of hyperon resonances has entered a new era of precision with advent of high-statistics photoproduction data from the CLAS detector at Jefferson Lab. These data have multi-particle final states, allowing clean identification of exclusive reactions associated with strange mesons and baryons. Examples of physics results are: evidence for isospin interference in the decay of the $\Lambda(1405)$ resonance; a strong suggestion of meson cloud effects in the structure of the $\Sigma(1385)$ resonance; data from $K^{*}$ photoproduction that will test the existence of the purported $K_{0}(800)$ meson. Properties of other hyperon resonances will also be studied in the near future.
\end{abstract}

Keywords: photoproduction, kaon, hyperon, resonance PACS: 13.30.Ce, 13.60.-r 14.20.Gk, 14.20.Jn

\section{INTRODUCTION}

The mass of the strange quark has a peculiar value, being close to the scale parameter of QCD, $\Lambda_{Q C D}[1]$. The light quarks $(u$ and $d$ ) have a mass small enough so that theoretical tools, such as chiral perturbation theory, can be used to describe the dynamics of these quarks. On the other hand, the heavy quarks ( $c, b$ and $t$ ) have a mass large enough so that non-relativistic potential theories, such as heavy quark effective theory (HQET), can be used for the dynamic of these quarks. The strange quark is too heavy for chiral dynamics and too light for HQET, which makes it difficult to describe theoretically [1].

Because of the theoretical difficulty to describe the dynamics of the strange quark, it is important to measure the properties of particles with non-zero strangeness in order to provide guidance for theoretical models. For example, it has been debated for some time [2] whether the structure of the first excited state of the $\Lambda$ baryon, the $\Lambda(1405)$ with $J^{P}=\frac{1}{2}^{-}$, is a $\bar{K} N$ bound state or a orbital excitation of the $u d s$ valence quarks in a conventional baryon quark model. The former would have strong 5-quark components in its wave-function, whereas the latter would have a predominantly 3-quark component. The fact that this issue is still not resolved is a testiment to the theoretical ambiguities in the dynamics of the strange quark.

Recently, the CLAS Collaboration has collected high-quality data for photoproduction of baryons with non-zero strangeness. The large statistics for these measurements forces constraints on theoretical models of strangeness production that were not possible previously. In particular, photoproduction of the ground state hyperons $\Lambda$ and $\Sigma$ shows evidence for a new nucleon resonance $\left(N^{*}\right)$ that couples strongly to strangeness decay, which may explain why it was not seen in partial wave analysis of pion scattering experiments. The search for $N^{*}$ resonances is important because significantly fewer $N^{*}$ 's are observed than are predicted by quark models [3], known as the "missing" resonances problem.

In addition to data on photoproduction of the ground state hyperons, data on hyperon resonances (with an associated kaon) and on kaon resonances (with a ground state hyperon) are now being released. These new data allow further investigations into the dynamics of the strange quark in mesons and baryons.

\section{CLAS DETECTOR AND RESULTS}

The CLAS detector, located at the Thomas Jefferson National Accelerator Facility, is a large acceptance spectrometer with a toroidal magnetic field [4]. Beams of electrons or photons (produced via bremsstrahlung) with energies up to 6 $\mathrm{GeV}$ are incident on target of hydrogen, deuterium, or nuclear targets at the center of CLAS. For photoproduction, the energy of the photon is given by a tagging detector which records the momentum of scattered electrons. Multi-particle final states are detected at CLAS with high rates, providing billions of triggered events. 
Results for the reaction $\gamma p \rightarrow K^{+} \Lambda$ measured at CLAS have been published [5] and are described elsewhere [6]. Of special interest, as mentioned above, is the observation of new $N^{*}$ resonances using both cross sections and polarization data. Curiously, the $\Lambda$ is polarized nearly $100 \%$ in the direction of circularly polarized photons [6] which was not predicted by any theoretical reaction model before the CLAS measurements. The precision of the CLAS data has sparked new theoretical interest in strangeness production.

The primary focus of the current report is on hyperon resonances. Perhaps one of the most interesting results in the past decade from CLAS measurements is for the reaction $\gamma p \rightarrow K^{+} \Lambda(1405)$, where the decays $\Lambda^{*} \rightarrow \Sigma^{+} \pi^{-}$and $\Lambda^{*} \rightarrow \Sigma^{-} \pi^{+}$were measured simultaneously. In a constituent quark model, where the $\Lambda(1405)$ is described as an orbital excitation of the ground-state $\Lambda(1115)$, these two decays should be isospin symmetric. However, in models where the $\Lambda(1405)$ is dynamically generated [7], interference between the $\bar{K} N$ and $\Sigma \pi$ poles can produce asymmetric decays. The Carnegie Melon University group at CLAS [8] measured dramatically different decays for the $\Lambda(1405) \rightarrow \Sigma \pi$, showing that this hyperon resonance has a more complex structure than predicted by simple quark models. This is the first time that clear isospin interference has been observed in the strong decay of a well established baryon resonance.

\section{$\Sigma^{*}(1385)$ Photoproduction}

Few data exist for photoproduction of the $\Sigma^{*}$ resonance, which is a partner to the well-known $\Delta$ resonance in the baryon decuplet. This is due in part to the difficulty of separating the $\Sigma^{* 0}$ at a mass of $1.385 \mathrm{GeV}$ from the $\Lambda(1405)$ resonance, which overlap due to their natural decay widths. Hence, the first differential cross sections with reasonable statistics were measured for $\Sigma^{*-}$ photoproduction from the neutron [9], where the neutral $\Lambda$ resonances are not seen. More precise measurements are being analyzed for $\Sigma^{*}$ photoproduction at CLAS, but are still very preliminary.

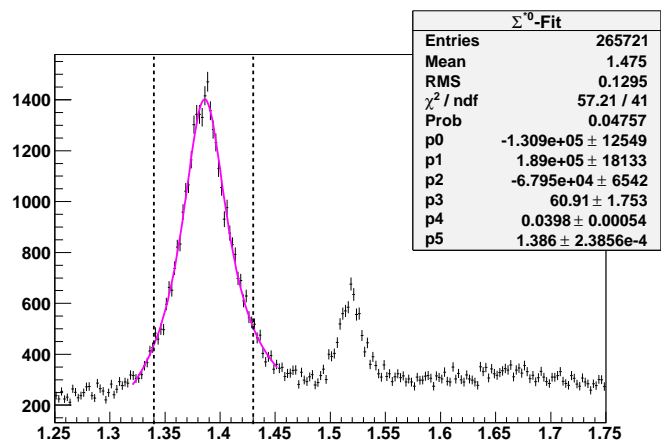

FIGURE 1. Missing mass (in $\mathrm{GeV}$ ) of the $K^{+}$for the reaction $\gamma p \rightarrow K^{+} \Lambda X$ showing peaks at the masses of the $\Sigma^{0}(1385)$ and $\Lambda(1520)$ resonances. The vertical scale shows counts.

One result of particular interest is the electromagnetic (EM) decay of the $\Sigma^{* 0}$ to the $\Lambda \gamma$ final state, which was published by CLAS [10]. The EM decay can be calculated exactly for a given theoretical model, and gives direct information on the wave-function of the baryon. Precise measurements have been made for the $\Delta \rightarrow N \gamma$ transition, yet no useful data were available for the $\Sigma^{*} \rightarrow Y \gamma$ transitions before the CLAS measurement.

Using a recent high-statistics data set, the $\Sigma^{* 0} \rightarrow \Lambda \gamma$ EM decay has been remeasured at CLAS, reducing the statistical uncertainty from about $30 \%$ to about $10 \%$. The smaller uncertainty is important, because effects from the pion cloud give a $\sim 30 \%$ increase in the theoretical prediction [11] for $\Delta \rightarrow N \gamma$ at low $Q^{2}$, which is necessary for agreement with data.

The published CLAS decay width for $\Sigma^{* 0} \rightarrow \Lambda \gamma$ is about a factor of two larger than theoretical predictions (for a range of theoretical models) [10], suggesting that meson cloud effects are significant. The new (preliminary) CLAS results for this EM decay width are consistent with the earlier results, but have much better precision. To show the quality of the new data, the missing mass off the $K^{+}$for the events of interest are shown in Fig. 1. Some background is seen under the $\Sigma^{* 0}$ peak at $1.385 \mathrm{GeV}$, which is mostly eliminated by a cut on the confidence level of a kinematic fit to the final state. The key step in the analysis is to separate the strong decay $\Sigma^{* 0} \rightarrow \Lambda \pi^{0}$ from the EM decay, which can be done reliably using kinematic fits.

Knowledge of the quark structure of the $\Sigma^{*}$ resonance is important, as it is the lowest-lying excited state of the strange baryons. Being a member of the baryon decuplet, along with the $\Delta$ resonance, it has spin $J=3 / 2$. In the simple quark model, the decuplet baryons are visualized as a purely symmetric spin state of three valence quarks. Relationships between the decuplet baryons can be calculated using isospin ( $I$-spin) and the analogue $U$-spin $S U(3)$ 
flavor symmetries. However, it is known that $S U(3)$ symmetries are broken, and the degree of symmetry breaking is something that experiments can measure. A broader program of measuring EM decays from a variety of the decuplet baryons with strangeness is in progress at CLAS, and will provide a window into the quark substructure of these baryons.
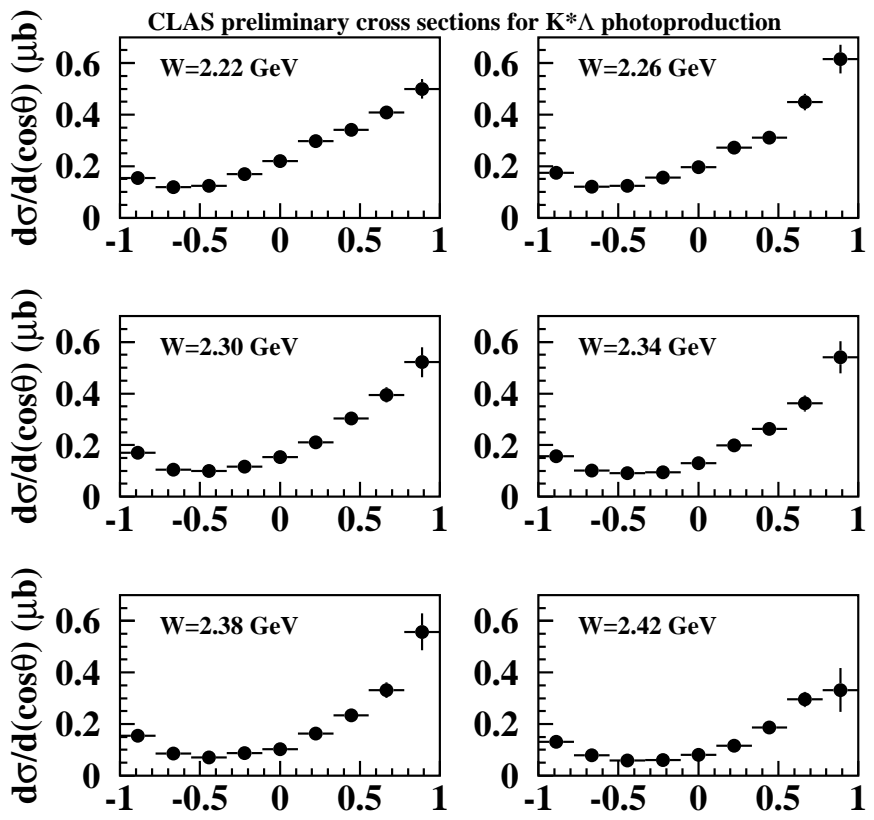

$\cos \theta$

$\cos \theta$

FIGURE 2. Preliminary angular distributions for photoproduction of the reaction $\gamma p \rightarrow K^{*+} \Lambda$ measured at CLAS.

\section{$K^{*} Y$ Photoproduction}

Before a recent CLAS publication [12], few data existed for photoproduction of the $K^{*}(892)$ vector meson, the spin-1 partner to the scalar kaon. The interest in $K^{*}$ photoproduction is, in part, to understand how spin is transferred from the photon to the strange quark. In the spin- 0 kaon, the polarization of the $\bar{s}$-quark can only be inferred through measurements of $\Lambda$ decay in the reaction $\gamma p \rightarrow K^{+} \Lambda$. As mentioned above, there appears to be nearly complete transfer of spin from a circularly polarized photon to the $\Lambda$. When the kaon is replaced by a $K^{*}$, the $K^{*}$ now carries some of the spin, providing new reaction dynamics.

Preliminary differential cross sections for the $\gamma p \rightarrow K^{*+} \Lambda$ reaction measured at CLAS are shown in Fig. 2. These angular distributions are for increasing photon energies near threshold, and have small statistical uncertainties (error bars are smaller than the symbols for most bins). These are the world's first high-statistics data for this reaction, and theorists are now developing models [13] for comparision with these data.

In addition to the $K^{*+}$ cross sections, preliminary results for the $\Lambda$ polarization from unpolarized beam show that there is less induced polarization compared with $K^{+} \Lambda$ photoproduction [5]. Results for $K^{*+} \Lambda$ production from polarized photons will be measured in the near future.

Because the $K^{*}$ is a vector meson, it is possible to construct the spin-density matrix elements for $K^{*+} \Lambda$ photoproduction. Preliminary results show substantial spin effects near threshold, similar to those seen for $\phi$-meson photoproduction. This suggests that significant spin transfer effects are seen to the $K^{*}$, as predicted by theory [13].

Within the context of a theoretical model, diagrams corresponding to $t$-channel exchange of virtual mesons show that the scalar meson, $K_{0}(800)$ with $J^{P}=0^{+}$, can contribute to the forward-angle cross sections and spin observables. The existence of the $K_{0}(800)$ (also called the kappa-meson) has been under discussion for many years. If it exists, it is 
probably the strange partner to the well established $a_{0}(980)$ and $f_{0}(980)$ mesons in a scalar nonet. The $K_{0}(800)$ is not seen directly as a peak in the $K \pi$ invariant mass, presumably due to its wide width (since it can easily "fall apart" into $K \pi$ because of its $J^{P}=0^{+}$spin-parity). A similar argument is made for the purported $\sigma$-meson, which would be the singlet member of this scalar nonet.

The $K_{0}(800)$ does not contribute to kaon photoproduction $t$-channel, so only data for $K^{*}$ photoproduction can support or weaken the case for the existence of the $K_{0}(800)$. So far, the case for the $K_{0}(800)$ is supported by the decay distributions of heavy mesons (such as $J / \psi \rightarrow \bar{K}^{* 0} K^{+} \pi^{-}$and $D^{+} \rightarrow K^{-} \pi^{+} \pi^{+}$, see [14]) but the evidence remains ambiguous. Data for linearly polarized beam for $K^{* 0}$ photoproduction, which has been measured at LEPS, will provide an unambiguous signal of the $K_{0}(800)$, if it exists. Similarly, the ratio of $K^{*+}$ and $K^{* 0}$ photoproduction total cross sections as a function of photon energy is predicted to be sensitive to the existence of the $K_{0}(800)$ meson. Analysis of these data are in progress.

\section{SUMMARY AND CONCLUSIONS}

The multi-particle data from high-statistics photon beams at CLAS have provided a rich source of results on hyperon resonance production. One example is the suggestion of a new nucleon resonance that couples strongly to the $K^{+} \Lambda$ final state, which was further identified by the study of spin-transfer to the $\Lambda$ from circularly polarized beam. Of particular note is the isospin interference effects seen in the decays of the $\Lambda(1405)$ hyperon resonance.

Models of the structure of hyperon resonances can be constrained by measurements of EM decays, which do not suffer from the ambiguities of strong decays. Although EM decays are much harder to measure, due to their small branching ratios, the results are more interesting because of the unambiguous predictions within a given quark model. Results for both $\Delta \rightarrow N \gamma$ and $\Sigma^{* 0} \rightarrow \Lambda \gamma$ are larger than simple quark model predictions. In the case of the $\Delta$, the pion cloud makes a significant enhancement to the EM transition amplitude [11]. Similar meson cloud effects are now being investigated for the $\Sigma^{*}$ EM decay.

For $K^{*}$ photoproduction, high-precision data are just now becoming available. Current theoretical models are phenomenological, and data are necessary to constrain the various coupling constants in the model. One unconstrained parameter is the contribution of the $K_{0}(800)$ scalar meson, which is not firmly established. The existence of the $K_{0}(800)$ would fill in the missing places in the meson nonet that includes the well established $a_{0}(980)$ and $f_{0}(980)$ mesons, and hence it is important to measure $K^{*}$ photoproduction reactions to see whether the data are described with diagrams that require $K_{0}(800)$ exchange in the $t$-channel.

\section{ACKNOWLEDGMENTS}

We gratefully acknowledge the CLAS Collaboration and the CEBAF accelerator staff, without which this work would not be possible. This work is supported in part by NSF grant PHY-0653454.

\section{REFERENCES}

1. R.L. Jaffe, International Workshop on Nuclear and Particle Physics at J-PARC, 2008.

2. J.W. Darewych, R. Koniuk and N. Isgur, Phys. Rev. D 32 (1985) 1765.

3. S. Capstick and W. Roberts, Phys. Rev. D 58 (1998) 074011.

4. B. Mecking et al., Nucl. Instr. Meth. A 503 (2003) 513.

5. M. McCracken et al., Phys. Rev. C 81 (2010) 025201.

6. R. Schumacher, Nucl. Phys. A 835 (2010) 231.

7. J.C. Nacher, E. Oset, H. Toki and A. Ramos, Phys. Lett. B 455 (1999) 55.

8. R. Schumacher and K. Moriya, these proceedings.

9. K. Hicks et al., Phys. Rev. Lett. 102 (2009) 012501.

10. S. Taylor et al., Phys. Rev. C 71 (2005) 054609.

11. B. Julià-Días, T.-S. H. Lee, T. Sato and L.C. Smith, ' Phys. Rev. C 75 (2007) 015205.

12. I. Hleiqawi et al., Phys. Rev. C 75 (2007) 042201; 76 (2007) 039905.

13. Y. Oh and H. Kim, Phys. Rev. C 74 (2006) 015208.

14. T. Komada (BES Collaboration), AIP Conf. Proc. 717 (2004) 337. 